\title{
UNIVALENCE OF HARMONIC FUNCTIONS, THE PROBLEM OF PONNUSAMY AND SAIRAM, AND CONSTRUCTIONS OF UNIVALENT POLYNOMIALS
}

\begin{abstract}
The criterion of the univalence of a harmonic mapping is obtained in this paper. Particularly, it permits to formulate the conjecture of coincidence of the harmonic function classes $S_{H}^{0}=S_{H}^{0}(S)$ (the problem of Ponnusamy and Sairam), in analytic form. The method of construction of the univalent harmonic polynomials with desired properties, according to a given harmonic function, is obtained by means of the univalence criteria.
\end{abstract}

Key words: harmonic functions, criteria of the univalence, harmonic univalent polynomials

2010 Mathematical Subject Classification: 30C55, 30C10, $31 \mathrm{A05}$

1. Introduction. Let $S$ be the class of all analytic and univalent functions of the form $f(z)=z+\sum_{n=2}^{\infty} a_{n} z^{n}$ in the disk $\Delta=\{z \in \mathbb{C}$ : $|z|<1\}$. The problem of determining a necessary coefficient condition in this class was set up by Bieberbach [1]. It consisted of validity of the inequality $\left|a_{n}\right| \leq n$ for each $n \in \mathbb{N}$ (equality for the Koebe function $k(z)=z /(1-z)^{2}$ and its rotations $\left.k_{\theta}(z)=e^{-i \theta} k\left(z e^{i \theta}\right)\right)$. The Bieberbach hypothesis contributed largely to the origin and development of a great number of ideas and methods in complex analysis. The full solution of this problem was finally given by de Branges [2].

The theory of univalent harmonic functions began its active development since the eighties of the previous century. Here the main object of research is the class $S_{H}$ of harmonic univalent and sense-preserving 
functions $f$ in $\Delta$ given by $f(z)=h(z)+\overline{g(z)}$, where

$$
h(z)=\sum_{n=1}^{\infty} a_{n} z^{n} \text { and } \overline{g(z)}=\sum_{n=1}^{\infty} a_{-n} \bar{z}^{n}
$$

S with $a_{1}=1$ (see, for example, [3]). The class $S_{H}$ is an analog of the class $S$. For obtaining information about the functions $S_{H}$ it is often convenient to have such information about the functions of the subclass $S_{H}^{0} \subset$ $\subset S_{H}$, where $S_{H}^{0}=\left\{f \in S_{H}: a_{-1}=0\right\}$. This circumstance explains the interest in studying $S_{H}^{0}$. In [4] Clunie and Sheil-Small formulated the following conjecture (the problem about coefficients in $S_{H}^{0}$ ): for all $f \in S_{H}^{0}$ and $n \in \mathbb{N}$, the inequalities

$$
\left|a_{n}\right| \leq \frac{(2 n+1)(n+1)}{6}, \quad\left|a_{-n}\right| \leq \frac{(2 n-1)(n-1)}{6}, \quad\left|a_{n}-a_{-n}\right| \leq n
$$

are true. A great deal of papers are dedicated to this conjecture. Particularly, in the paper of Ponnusamy and Sairam Kaliraj [5] this conjecture, together with some other results was proved for the subclass

$$
S_{H}^{0}(S)=\left\{f=h+\bar{g} \in S_{H}^{0}: h+e^{i \phi} g \in S \text { for some } \phi \in \mathbb{R}\right\}
$$

of $S_{H}^{0}$. Besides, in this paper the authors conjectured that $S_{H}^{0}(S)=S_{H}^{0}$, whose prove would permit us to obtain the full solution of the coefficients problems (1) of Clunie and Sheil-Small. In this paper the criterion of univalency of harmonic functions (Theorem 1) is obtained. With the aid of this, the criterion for functions belonging to $S_{H}^{0}(S)$ (Theorem 2) is obtained and several examples are exhibited. Theorem 3 permits to construct harmonic univalent polynomials for a given $f \in S_{H}$.

\section{The univalence criterion and the conjecture of hypothesis} of Ponnusamy and Sairam Kaliraj. The univalence criterion of an arbitrary harmonic function in $\Delta$ of the form

$$
f(z)=\sum_{n=1}^{\infty}\left(a_{n} z^{n}+a_{-n} \bar{z}^{n}\right)
$$

will be obtained by analogy to Bazilevich's [6] univalence criterion for analytic functions: 
Theorem A. [6] An analytic function $f(z)=\sum_{n=1}^{\infty} a_{n} z^{n}$ in $\Delta$ is univalent in $\Delta$ if and only if for each $z \in \Delta$ and each $t \in[0, \pi / 2]$,

$$
\sum_{n=1}^{\infty} a_{n} \frac{\sin n t}{\sin t} z^{n-1} \neq 0,\left.\quad\left(\frac{\sin n t}{\sin t}\right)\right|_{t=0}=n
$$

Theorem 1. Harmonic sense-preserving function in $\Delta$, determined by the formula (2), is univalent in $\Delta$ if and only if for each $z \in \Delta \backslash\{0\}$ and each $t \in(0, \pi / 2]$,

$$
\sum_{n=1}^{\infty}\left[\left(a_{n} z^{n}-a_{-n} \bar{z}^{n}\right) \frac{\sin n t}{\sin t}\right] \neq 0
$$

Proof. Let $f \in S_{H}$. Then, for $z_{1}, z_{2}\left(z_{1} \neq z_{2}\right)$ from $\Delta$, we have

$$
\frac{f\left(z_{2}\right)-f\left(z_{1}\right)}{z_{2}-z_{1}} \neq 0
$$

Particularly, for $z_{1}=r e^{i \theta_{1}} \neq r e^{i \theta_{2}}=z_{2}, r \in(0,1), \theta_{k} \in \mathbb{R}$, this is equivalent to

$$
\begin{gathered}
\frac{f\left(r e^{i \theta_{2}}\right)-f\left(r e^{i \theta_{1}}\right)}{r e^{i \theta_{2}}-r e^{i \theta_{1}}}= \\
=\sum_{n=1}^{\infty} r^{n-1}\left(a_{n} \frac{e^{i n \theta_{2}}-e^{i n \theta_{1}}}{e^{i \theta_{2}}-e^{i \theta_{1}}}+a_{-n} \frac{e^{-i n \theta_{2}}-e^{-i n \theta_{1}}}{e^{i \theta_{2}}-e^{i \theta_{1}}}\right) \neq 0 .
\end{gathered}
$$

Without loss of generality, we may assume that $\theta_{1}<\theta_{2} \leq \theta_{1}+\pi$. Let

$$
t=\frac{\theta_{2}-\theta_{1}}{2} \in(0, \pi / 2] \text { and } \theta=\frac{\theta_{2}+\theta_{1}}{2} \in \mathbb{R} .
$$

Then

$$
\frac{e^{i n \theta_{2}}-e^{i n \theta_{1}}}{e^{i \theta_{2}}-e^{i \theta_{1}}}=e^{i(n-1) \frac{\theta_{2}+\theta_{1}}{2}} \frac{e^{i n \frac{\theta_{2}-\theta_{1}}{2}}-e^{-i n \frac{\theta_{2}-\theta_{1}}{2}}}{e^{i \frac{\theta_{2}-\theta_{1}}{2}}-e^{-i \frac{\theta_{2}-\theta_{1}}{2}}}=e^{i(n-1) \theta} \frac{e^{i n t}-e^{-i n t}}{e^{i t}-e^{-i t}},
$$

and

$$
\frac{e^{-i n \theta_{2}}-e^{-i n \theta_{1}}}{e^{i \theta_{2}}-e^{i \theta_{1}}}=
$$




$$
=\overline{\left(\frac{e^{i n \theta_{2}}-e^{i n \theta_{1}}}{e^{i \theta_{2}}-e^{i \theta_{1}}}\right)} \frac{e^{-i \theta_{2}}-e^{-i \theta_{1}}}{e^{i \theta_{2}}-e^{i \theta_{1}}}=e^{-i(n-1) \theta} \frac{e^{i n t}-e^{-i n t}}{e^{i t}-e^{-i t}}\left(-e^{-2 i \theta}\right) .
$$

Hence (4) may be represented as

$$
\sum_{n=1}^{\infty}\left[\left(a_{n} r^{n-1} e^{i(n-1) \theta}-a_{-n} r^{n-1} e^{-i(n-1) \theta} e^{-2 i \theta}\right) \frac{\sin n t}{\sin t}\right] \neq 0
$$

which is equivalent to

$$
\sum_{n=1}^{\infty}\left[\left(a_{n} z^{n-1}-a_{-n} \bar{z}^{n-1} e^{-2 i \theta}\right) \frac{\sin n t}{\sin t}\right] \neq 0
$$

where $z=r e^{i \theta} \in \Delta, \theta=\arg z, t \in(0, \pi / 2]$. But (5) $\Longleftrightarrow(3)$. Let us note, that (5) is fulfilled for $z=0$ as well, because $\left|a_{1}\right|>\left|a_{-1}\right|$, since $f$ is sense-preserving.

Let us next prove the inverse proposition. Suppose a harmonic function (2) is sense-preserving in $\Delta$ and condition (3) is fulfilled. According to the accepted designations this is equivalent to fulfilling of the condition $(4)$, i. e.

$$
\frac{f\left(z_{2}\right)-f\left(z_{1}\right)}{z_{2}-z_{1}} \neq 0 \quad \forall z_{1}=r e^{i \theta_{1}} \neq r e^{i \theta_{2}}=z_{2}, r \in(0,1), \theta_{k} \in \mathbb{R} .
$$

Thus $f$ is univalent on any circle $\{z \in \mathbb{C}:|z|=r\}$.

The local univalence of the function $f$ implies that $\partial f\left(\Delta_{r}\right) \subset f\left(\partial \Delta_{r}\right)$, where $\Delta_{r}=\{z \in \mathbb{C}:|z|<r\}$. Then the assumption that $f$ is not univalent (but locally univalent) in $\Delta$ implies the existence of a disk $\Delta_{R}$ (a disk of $f$ univalence), in which $f$ is univalent, but on $\partial \Delta_{R}$ there exist points $z_{1} \neq z_{2}$ such that $f\left(z_{1}\right)=f\left(z_{2}\right)$. This contradicts the univalence of $f$ on the circle $\{z \in \mathbb{C}:|z|=R\}$. This contradiction proves Theorem 1 .

The following theorem represents the criterion of belonging of a function to the class $S_{H}^{0}(S)$.

Theorem 2. Let $f \in S_{H}^{0}$. Define

$$
A=A(z, t)=\sum_{n=1}^{\infty} a_{n} \frac{\sin n t}{\sin t} z^{n}, \quad B=B(z, t)=\sum_{n=1}^{\infty} \overline{a_{-n}} \frac{\sin n t}{\sin t} z^{n},
$$

and

$$
E=\{(z, t) \in(\Delta \backslash\{0\}) \times(0, \pi / 2]:|A(z, t)|=|B(z, t)|\} .
$$


Then $f \in S_{H}^{0}(S)$ if and only if there exists a $\phi \in[0,2 \pi)$ such that

$$
A(z, t) \neq-e^{i \phi} B(z, t) \quad \forall(z, t) \in E .
$$

Proof. Let $f=h+\bar{g} \in S_{H}^{0}(S)$ and let it be determined by (2). According to the definition, $S_{H}^{0}(S) \ni f$ if and only if there exists a $\phi \in[0,2 \pi)$ such that $h+e^{i \phi} g \in S$. Theorem A implies that

$$
\begin{gathered}
\sum_{n=1}^{\infty}\left(a_{n}+e^{i \phi \overline{a_{-n}}}\right) \frac{\sin n t}{\sin t} z^{n} \neq 0, \text { for } z \in \Delta \backslash\{0\}, t \in(0, \pi / 2], \\
\Longleftrightarrow A(z, t) \neq-e^{i \phi} B(z, t) \quad \forall(z, t) \in E .
\end{gathered}
$$

This completes the proof.

Corollary 1. Let $f \in S_{H}^{0}$ and $E$ be the set defined in Theorem 2. If $E=\emptyset$, then $f \in S_{H}^{0}(S)$.

Remark 1. Denote $q(z)=\sum_{n=1}^{\infty} \frac{\sin n t}{\sin t} z^{n}$. Then

$$
A(z, t)=\frac{1}{2 \pi i} \int_{|\zeta|=\rho} h\left(\frac{z}{\zeta}\right) q(\zeta) \frac{d \zeta}{\zeta}, B(z, t)=\frac{1}{2 \pi i} \int_{|\zeta|=\rho} g\left(\frac{z}{\zeta}\right) q(\zeta) \frac{d \zeta}{\zeta} .
$$

Hence the statement of Theorem 2 may be represented by means of these integrals.

Example. For a fixed $R \in(0,1)$, consider the harmonic function $f=h+\bar{g}$ in $\Delta$, where

$$
h(z)=\frac{z}{(1-R z)^{2}} \text { and } g(z)=k z^{2}, \quad k \in \mathbb{R} .
$$

By means of Theorem 1 we determine for which values of $k$ and $R, f \in S_{H}^{0}$. Applying Theorem 2, let us show that for these values of parameters, the function $f \in S_{H}^{0}(S)$.

Firstly we need to determine, for which values of the parameters, the function $f$ is sense-preserving in $\Delta$. The condition of sense-preservation means the validity for any $z \in \Delta$ of the inequality

$$
\left|h^{\prime}\right|-\left|g^{\prime}\right|=\left|\frac{1+R z}{(1-R z)^{3}}\right|-|2 k z|>0
$$


which holds if

$$
\min _{|z|=r}\left|\frac{1+R z}{(1-R z)^{3}}\right|=\frac{1-R r}{(1+R r)^{3}}>2|k| r, \forall r \in[0,1) .
$$

Since the function $\frac{1-R r}{r(1+R r)^{3}}$ decreases with respect to $r$, the latter inequality gives

$$
2|k| \leq \frac{1-R}{(1+R)^{3}}
$$

The values of parameters for which the function $f$ is sense-preserving in $\Delta$ is determined from (6). Further, let the condition (6) be valid. According to Theorem 1, $f$ is univalent if and only if $A(z, t) \neq \overline{B(z, t)}$ (designations from Theorem 2) in $(\Delta \backslash\{0\}) \times(0, \pi / 2]$. Let us show that the equality $A(z, t)=\overline{B(z, t)}$ is not possible. We see that

$$
\begin{aligned}
A(z, t) & =\sum_{n=1}^{\infty} n R^{n-1} z^{n} \frac{e^{i n t}-e^{-i n t}}{2 i \sin t}= \\
& =\frac{1}{2 i \sin t}\left[\frac{z e^{i t}}{\left(1-R z e^{i t}\right)^{2}}-\frac{z e^{-i t}}{\left(1-R z e^{-i t}\right)^{2}}\right]= \\
& =\frac{z\left(1-R^{2} z^{2}\right)}{\left(1-2 R z \cos t+R^{2} z^{2}\right)^{2}}
\end{aligned}
$$

and

$$
B(z, t)=2 \bar{k} z^{2} \cos t
$$

Now we show that for $t \in(0, \pi / 2)$ and $z \in \Delta \backslash\{0\}$, the equation

$$
\frac{z\left(1-R^{2} z^{2}\right)}{\left(1-2 R z \cos t+R^{2} z^{2}\right)^{2}}=2 k \bar{z}^{2} \cos t
$$

has no solution. It is sufficient to show that in (7) the absolute values of the left hand and right hand sides are not equal. If

$$
|2 k|=\left|\frac{1-R^{2} z^{2}}{z \cos t\left(1-2 R \cos t z+R^{2} z^{2}\right)^{2}}\right|=L(z, t),
$$

then for some $t$,

$$
|2 k| \geq \min _{0<|z| \leq 1} L(z, t)=\min _{|z|=1} L(z, t)=L(-1, t)=
$$




$$
=\frac{1-R^{2}}{\cos t\left(1+2 R \cos t+R^{2}\right)^{2}}>\frac{1-R}{(1+R)^{3}}
$$

for values of $t$ under consideration. Thus a contradiction with (6) is obtained. Therefore, if the condition (6) is fulfilled, then all functions $f$ from this example are univalent. As shown above, $|A(z, t)| \neq|B(z, t)|$, in $(\Delta \backslash\{0\}) \times(0, \pi / 2]$ and therefore the set $E$ defined in Theorem 2 is empty. Hence, for parameters' values satisfying the inequality (6), $f \in S_{H}^{0}(S)$.

3. Univalent harmonic polynomials are functions of the form $P=h+\bar{g}$, where $h$ and $g$ are classic polynomials in $z$. Generally speaking, there is not much information about univalent harmonic polynomials than about other functions from $S_{H}$ (here we speak about univalence in $\Delta$ ) (see $[7])$.

For example, in a survey paper [8] the authors note: "Finding a method of constructing sense-preserving univalent harmonic polynomials is another important problem". In the analytic case, Bazilevich [6] proposed a method of construction of univalent polynomials, associated with a given function from $S$. Further, his idea has been transferred to harmonic case in Theorem 3. Moreover, unlike with analytic case, the proof will be constructive. Thus, Theorem 3 gives an opportunity to construct harmonic univalent polynomials of sufficiently high power for any function $f \in S_{H}^{0}$.

Lemma 1. If $f \in S_{H}^{0}, r \in(0,1), \quad t, \phi \in \mathbb{R}$, then

$$
\left|\frac{f\left(r e^{i t}\right)-f\left(r e^{i \phi}\right)}{r e^{i t}-r e^{i \phi}}\right| \geq \frac{1-r}{4 \alpha r}\left(\frac{1-r}{1+r}\right)^{\alpha}\left[1-\left(\frac{1-r}{1+r}\right)^{2 \alpha}\right],
$$

where $\alpha\left(=\operatorname{ord}_{H}\right) \stackrel{\text { def }}{=} \sup _{f \in S_{H}}\left|a_{2}\right|$.

Proof. If $f=h+\bar{g} \in S_{H}^{0}$, then the linear invariance of the class $S_{H}$ (see [9]) implies that

$$
F(z)=\frac{f\left(\frac{z+a}{1+\bar{a} z}\right)-f(a)}{h^{\prime}(a)\left(1-|a|^{2}\right)} \in S_{H} \quad \forall a \in \Delta .
$$

Let

$$
a=r e^{i \phi}, \quad \frac{z+a}{1+\bar{a} z}=r e^{i t}, \quad \text { i. e. } \quad z=\frac{r e^{i t}-a}{1-\bar{a} r e^{i t}} .
$$


From the affine invariance (see. [9]) of the class $S_{H}$, it follows that the function

$$
\psi(z)=\frac{F(z)-a_{-1} \overline{F(z)}}{1-\left|a_{-1}\right|^{2}} \in S_{H}^{0}, \quad \text { where } a_{-1}=\frac{\partial F}{\partial \bar{z}}(0)=\frac{\overline{g^{\prime}(a)}}{h^{\prime}(a)} .
$$

Hence,

$$
|F(z)|\left(1+\left|a_{-1}\right|\right) \geq\left|F(z)-a_{-1} \overline{F(z)}\right| \geq|\psi(z)|\left(1-\left|a_{-1}\right|^{2}\right),
$$

so that $|F(z)| \geq|\psi(z)|\left(1-\left|a_{-1}\right|\right)$. Since $f \in S_{H}^{0}$, then $\frac{\partial f}{\partial \bar{z}}(0)=0$, i. e. $g^{\prime}(0)=0$. Since $f$ is sense-preserving in $\Delta$, we have $\left|g^{\prime}(z) / h^{\prime}(z)\right|<1$ in $\Delta$. Therefore, according to Schwarz's lemma, $\left|a_{-1}\right|=\left|g^{\prime}(a) / h^{\prime}(a)\right| \leq r$ and

$$
|F(z)| \geq|\psi(z)|(1-r) .
$$

For any function $\psi=H+\bar{G} \in S_{H}^{0}$ and any $z \in \Delta$, one has [9]:

$$
\frac{1}{2 \alpha}\left[1-\left(\frac{1-|z|}{1+|z|}\right)^{\alpha}\right] \leq|\psi(z)| \text { and } \frac{(1-|z|)^{\alpha-1}}{(1+|z|)^{\alpha+1}} \leq\left|H^{\prime}(z)\right|
$$

from which with regard to (8) we obtain

$$
\begin{gathered}
\left|f\left(r e^{i t}\right)-f\left(r e^{i \phi}\right)\right| \geq \frac{1}{2 \alpha}\left[1-\left(\frac{1-|z|}{1+|z|}\right)^{\alpha}\right](1-r)\left(1-r^{2}\right)\left|h^{\prime}\left(r e^{i \phi}\right)\right| \geq \\
\geq \frac{1-r}{2 \alpha}\left(\frac{1-r}{1+r}\right)^{\alpha}\left[1-\left(\frac{1-|z|}{1+|z|}\right)^{\alpha}\right]
\end{gathered}
$$

where

$$
z=\frac{r\left(e^{i t}-e^{i \phi}\right)}{1-r^{2} e^{i(t-\phi)}}
$$

Denote by $w=e^{i s}=e^{i(t-\phi)}$ and $\zeta=\frac{1-w}{1-r^{2} w}$. Then

$$
w=\frac{1-\zeta}{1-r^{2} \zeta}, \quad 0 \leq|z|=r|\zeta| \leq \frac{2 r}{1+r^{2}}, \quad|1-w|=\frac{|\zeta|\left(1-r^{2}\right)}{\left|1-r^{2} \zeta\right|}
$$

Represent (9) as follows

$$
\left|\frac{f\left(r e^{i t}\right)-f\left(r e^{i \phi}\right)}{r e^{i t}-r e^{i \phi}}\right| \geq \frac{1-r}{2 \alpha}\left(\frac{1-r}{1+r}\right)^{\alpha}\left[1-\left(\frac{1-r|\zeta|}{1+r|\zeta|}\right)^{\alpha}\right] \frac{\left|1-r^{2} \zeta\right|}{r|\zeta|\left(1-r^{2}\right)} \geq
$$




$$
\geq \frac{1}{2 \alpha}\left(\frac{1-r}{1+r}\right)^{\alpha}\left[1-\left(\frac{1-x}{1+x}\right)^{\alpha}\right] \frac{1-r x}{x(1+r)},
$$

$x=r|\zeta| \in\left[0,2 r /\left(1+r^{2}\right)\right]$.

Define

$$
u(x)=\frac{1}{x}\left[1-\left(\frac{1-x}{1+x}\right)^{\alpha}\right], \quad x \in\left[0, \frac{2 r}{1+r^{2}}\right] .
$$

We show that $u$ is decreasing on $\left(0,2 r /\left(1+r^{2}\right)\right)$. Then,

$$
\begin{gathered}
x^{2} u^{\prime}(x) \leq 0 \Longleftrightarrow\left(\frac{1-x}{1+x}\right)^{\alpha-1} \frac{2 \alpha x}{(1+x)^{2}}-1+\left(\frac{1-x}{1+x}\right)^{\alpha} \leq 0 \\
\Longleftrightarrow 2 \alpha x+1-x^{2} \leq\left(1-x^{2}\right)\left(\frac{1+x}{1-x}\right)^{\alpha} \Longleftrightarrow \\
\Longleftrightarrow u_{1}(x) \leq u_{2}(x),
\end{gathered}
$$

where

$u_{1}(x)=\ln \left(1+2 \alpha x-x^{2}\right)$ and $u_{2}(x)=\alpha(\ln (1+x)-\ln (1-x))+\ln \left(1-x^{2}\right)$.

Note that $u_{1}(0)=0=u_{2}(0)$ and

$$
u_{1}^{\prime}(x)=\frac{2(\alpha-x)}{1+2 \alpha x-x^{2}} \leq \frac{2(\alpha-x)}{1-x^{2}}=u_{2}^{\prime}(x),
$$

since (see [9]) $3 \leq \alpha<48,9$. This proves the validity of (11) and hence $u(x)$ is decreasing on $\left(0,2 r /\left(1+r^{2}\right)\right)$ and hence

$$
\min _{x \in\left[0,2 r /\left(1+r^{2}\right)\right]} u(x)=u\left(\frac{2 r}{1+r^{2}}\right)=\frac{1+r^{2}}{2 r}\left[1-\left(\frac{1-r}{1+r}\right)^{2 \alpha}\right] .
$$

Then from (10) we obtain the desired inequality in Lemma 1. The proof of the lemma is complete.

In Lemma 2 below the estimates of coefficients of functions from $S_{H}^{0}$ will be obtained. The estimates are not exact, but they are sufficient to achieve the goal which we set up in this section (see Theorem 3).

Lemma 2. If $\alpha=\operatorname{ord} S_{H}, S_{H}^{0} \ni f$ with series expansion (2), then the following inequality is true:

$$
\left|a_{ \pm n}\right|<\frac{\left(2 e^{2}\right)^{\alpha}}{2 \alpha} n^{\alpha}, n \in \mathbb{N}
$$


Proof. Sheil-Small [9] proved for $f \in S_{H}^{0}$ the inequality

$$
|f(z)| \leq \frac{1}{2 \alpha}\left[\left(\frac{1+|z|}{1-|z|}\right)^{\alpha}-1\right], z \in \triangle \text {. }
$$

Hence for $n \in \mathbb{N}$ and $r \in(0,1)$,

$$
\left|a_{-n}\right|=\left|\frac{1}{2 \pi i} \int_{|z|=r} \frac{f(z) d \bar{z}}{\bar{z}^{n+1}}\right| \leq \frac{1}{2 \alpha r^{n}}\left(\frac{1+r}{1-r}\right)^{\alpha}=\frac{\psi(r)}{2 \alpha} .
$$

The same estimate is also true for $\left|a_{n}\right|, n \in \mathbb{N}$. In order to find a minimum of the right hand side of inequality we find the point of minimum of the function $\ln \psi(r)$ :

$$
(\ln \psi(r))^{\prime}=\frac{2 \alpha}{1-r^{2}}-\frac{n}{r}=0 \Longleftrightarrow r^{2}+\frac{2 \alpha}{n} r-1=0 .
$$

Therefore, $r_{0}=\sqrt{\alpha^{2} / n^{2}+1}-\alpha / n$ is the point of minimum and

$$
\left|a_{n}\right| \leq \frac{\psi\left(r_{0}\right)}{2 \alpha} \text { for all integer } n,
$$

where

$$
\begin{gathered}
\psi\left(r_{0}\right)= \\
=\left(\sqrt{\frac{\alpha^{2}}{n^{2}}+1}+\frac{\alpha}{n}\right)^{n}\left[\frac{n}{2 \alpha}\left(1+\sqrt{\frac{\alpha^{2}}{n^{2}}+1}+\frac{\alpha}{n}\right)\left(1+\sqrt{\frac{\alpha^{2}}{n^{2}}+1}-\frac{\alpha}{n}\right)\right]^{\alpha} .
\end{gathered}
$$

Using inequality $\sqrt{1+x} \leq 1+\sqrt{x}, x>0$, we obtain

$$
\begin{aligned}
& \psi\left(r_{0}\right) \leq\left(\frac{n}{\alpha}\right)^{\alpha}\left[\left(1+\frac{2 \alpha}{n}\right)^{\frac{n}{2 \alpha}}\right]^{2 \alpha}\left(2+\frac{\alpha}{n}\right)^{\alpha}= \\
& =(2 n)^{\alpha}\left(\frac{1}{2 n}+\frac{1}{\alpha}\right)^{\alpha}\left[\left(1+\frac{2 \alpha}{n}\right)^{n /(2 \alpha)}\right]^{2 \alpha} .
\end{aligned}
$$

Introduce

$$
\Psi(y)=y \ln \left(1+\frac{1}{y}\right)
$$


Then $\sup _{y>0} \Psi(y)=1$, since $\lim _{y \rightarrow+0} \Psi(y)<1, \lim _{y \rightarrow+\infty} \Psi(y)=1$, and, if $\Psi(y)$ has a maximum on the interval $(0, \infty)$ at the point $y_{0}$, then $\Psi^{\prime}\left(y_{0}\right)=0$. This gives

$$
\ln \left(1+\frac{1}{y_{0}}\right)=\frac{1}{1+y_{0}}
$$

which implies that

$$
\Psi\left(y_{0}\right)=\frac{y_{0}}{1+y_{0}}<1 .
$$

Hence from (12) we have

$$
\psi\left(r_{0}\right)<(2 n)^{\alpha} e^{2 \alpha} \text { and }\left|a_{ \pm n}\right|<\frac{\left(2 e^{2}\right)^{\alpha}}{2 \alpha} n^{\alpha}
$$

and the proof of Lemma 2 is complete.

Theorem 3. Let $f \in S_{H}^{0}$ and have the series expansion (1), $\alpha=$ $=\operatorname{ord} S_{H}, s=[\alpha+2]$, where [.] denotes the integer part of a number. Let $m \in \mathbb{N}, \epsilon \in\left(0,\left(2 e^{2}\right)^{\alpha} /(2 \alpha)\right)$ and

$$
r \in\left(\left[1-\frac{2 \alpha \epsilon}{\left(2 e^{2} m\right)^{\alpha}}\right]^{1 / m}, 1\right)
$$

let $N \in \mathbb{N}$ be so large that

$$
\begin{aligned}
& \frac{2}{|\ln r|}\left[\alpha(2+\ln 2)+\ln (s !)-(s+1) \ln |\ln r|-\ln (1-r)-\alpha \ln \frac{1-r}{1+r}+\right. \\
& \left.+\ln 4-\ln \left[1-\left(\frac{1-r}{1+r}\right)^{2 \alpha}\right]\right]+2<N, \quad m<N, \quad \frac{\ln N}{N} \leq \frac{|\ln r|}{2[\alpha+2]} .
\end{aligned}
$$

Then the harmonic polynomial

$$
P(z)=\sum_{k=1}^{N}\left(c_{k} z^{k}+c_{-k} \bar{z}^{k}\right)=\sum_{k=1}^{N}\left(a_{k} r^{k} z^{k}+a_{-k} r^{k} \bar{z}^{k}\right)
$$

is univalent in $\Delta$ and moreover $\left|a_{ \pm k}-c_{ \pm k}\right|<\epsilon$ for all $k=1, \ldots, m$.

Proof. Let us note that $2 \alpha \epsilon /\left(2 e^{2}\right)^{\alpha}<1$ for the indicated values $\epsilon$. Hence $\frac{2 \alpha \epsilon}{\left(2 e^{2} k\right)^{\alpha}}<1$ for $k=1, \ldots, m$. 
The function $\phi(x)=\left(1-x^{\alpha}(2 \alpha \epsilon) /\left(2 e^{2}\right)^{\alpha}\right)^{x}$ decreases on $(0,1]$, since $\frac{d \ln \phi(x)}{d x}<0$ for $x \in(0,1]$. Therefore, $\phi(1 / k) \leq \phi(1 / m)$ for each $k=$ $=1, \ldots, m$, and (13) implies the inequality $\phi(1 / k) \leq \phi(1 / m)<r$. Hence

$$
\left(1-r^{k}\right) \frac{\left(2 e^{2} k\right)^{\alpha}}{2 \alpha}<\epsilon \text { for } k=1, \ldots, m .
$$

From these inequalities and Lemma 2 we obtain inequalities for the first coefficients $c_{ \pm k}=r^{k} a_{ \pm k}$ of the polynomial $P(z)$ :

$$
\left|a_{ \pm k}-c_{ \pm k}\right|=\left|a_{ \pm k}-r^{k} a_{ \pm k}\right|<\epsilon, \quad k=1, \ldots, m .
$$

Let us verify, by the method of mathematical induction, that

$$
|\sin n t| \leq n \sin t \forall t \in(0, \pi / 2] \text { and } \forall n \in \mathbb{N} .
$$

The inequality is true for $n=1$. Assume that the inequality is true for $(n-1)$. Then

$$
\begin{gathered}
|\sin n t| \leq|\sin (n-1) t| \cos t+|\cos (n-1) t| \sin t \leq \\
\quad \leq \sin t[(n-1) \cos t+|\cos (n-1) t|] \leq n \sin t .
\end{gathered}
$$

Let us show under the hypothesis of the theorem that the polynomial $P(z)$ is univalent in $\triangle$. For $z \in \Delta$, let us estimate the remainder $R_{N}$ of the series. We have

$$
\left|R_{N}\right|=\left|\sum_{n=N+1}^{\infty}\left[\left(a_{n} z^{n}-a_{-n} \bar{z}^{n}\right) r^{n} \frac{\sin n t}{\sin t}\right]\right| \leq \frac{\left(2 e^{2}\right)^{\alpha}}{\alpha} \sum_{n=N+1}^{\infty} n^{\alpha+1} r^{n}
$$

according to Lemma 2. By hypothesis, $N|\ln r| \geq 2 s \ln N>\alpha+1$, and therefore, $N>\frac{\alpha+1}{|\ln r|}$. But for $x \in\left[\frac{\alpha+1}{|\ln r|}, \infty\right)$, the function $T(x)=$ $=x^{\alpha+1} r^{x}$ decreases. Hence with the increasing values of $n$, the terms of the series $\sum_{n=N}^{\infty} n^{\alpha+1} r^{n}$ decrease and therefore,

$$
\sum_{n=N+1}^{\infty} n^{\alpha+1} r^{n}<\int_{N}^{\infty} T(x) d x \leq \int_{N}^{\infty} x^{s} r^{x} d x .
$$


Integrating by parts successfully $s$ times, we obtain

$$
\int_{N}^{\infty} x^{s} r^{x} d x=\frac{N^{s} r^{N}}{|\ln r|}+\frac{s N^{s-1} r^{N}}{|\ln r|^{2}}+\frac{s(s-1) N^{s-2} r^{N}}{|\ln r|^{3}}+\cdots+\frac{s ! r^{N}}{|\ln r|^{s+1}} .
$$

Let us represent the condition $N|\ln r| \geq 2 s \ln N$ of the theorem as $r^{\frac{N}{2}} N^{s} \leq$ $\leq 1$. Hence $r^{N} N^{j} \leq r^{N / 2}$ for any $j=1, \ldots, s$. Taking into account these inequalities, we have the estimate

$$
\begin{gathered}
\int_{N}^{\infty} x^{s} r^{x} d x \leq \\
\leq \frac{s ! r^{N / 2}}{|\ln r|^{s+1}}\left[\frac{|\ln r|^{s}}{s !}+\frac{|\ln r|^{s-1}}{(s-1) !}+\ldots+\frac{|\ln r|}{1 !}+r^{N / 2}\right]<\frac{r^{N / 2-1} s !}{|\ln r|^{s+1}} .
\end{gathered}
$$

Hence

$$
\left|R_{N}\right| \leq \frac{\left(2 e^{2}\right)^{\alpha}}{\alpha} \frac{r^{N / 2-1} s !}{|\ln r|^{s+1}} .
$$

From (14), Theorem 1 and Lemma 1 we obtain

$$
\begin{gathered}
\left|\sum_{n=1}^{N}\left[\left(a_{n} z^{n}-a_{-n} \bar{z}^{n}\right) r^{n} \frac{\sin n t}{\sin t}\right]\right| \geq \\
\geq\left|\sum_{n=1}^{\infty}\left[\left(a_{n} z^{n}-a_{-n} \bar{z}^{n}\right) r^{n} \frac{\sin n t}{\sin t}\right]\right|-\left|R_{N}\right| \geq \\
\geq \frac{1-r}{4 \alpha}\left(\frac{1-r}{1+r}\right)^{\alpha}\left[1-\left(\frac{1-r}{1+r}\right)^{2 \alpha}\right]-\frac{\left(2 e^{2}\right)^{\alpha}}{\alpha} \frac{r^{N / 2-1} s !}{|\ln r|^{s+1}}=Q .
\end{gathered}
$$

If now $Q>0$, then the univalency of the polynomial $P$ follows from Theorem 1. The latter inequality is equivalent to

$$
\begin{gathered}
\frac{2}{|\ln r|}\left[\alpha(2+\ln 2)+\ln (s !)-(s+1) \ln |\ln r|-\ln (1-r)-\alpha \ln \frac{1-r}{1+r}+\right. \\
\left.+\ln 4-\ln \left[1-\left(\frac{1-r}{1+r}\right)^{2 \alpha}\right]\right]+2<N
\end{gathered}
$$

which proves Theorem 3 .

The proof of Theorem 3 implies the following result. 
Corollary 2. Let $f \in S_{H}^{0}$ and $f$ have the expansion (1), $\alpha=\operatorname{ord} S_{H}$, $r \in(0,1)$ and $0 \neq m \in \mathbb{Z}$. Then the $m$-th coefficient $c_{m}=a_{m} r^{|m|}$ of the univalent function

$$
f(r z)=\sum_{n=1}^{\infty}\left(c_{n} z^{n}+c_{-n} \bar{z}^{n}\right)
$$

may be replaced by $c_{m}+\lambda$, where $\lambda \in \mathbb{C}$, and

$$
|\lambda|<\frac{1-r}{4 \alpha|m|}\left(\frac{1-r}{1+r}\right)^{\alpha}\left[1-\left(\frac{1-r}{1+r}\right)^{2 \alpha}\right]
$$

without loss of univalence.

Proof. According to Lemma 1,

$$
\left|\sum_{n=1}^{\infty}\left[\left(a_{n} z^{n}-a_{-n} \bar{z}^{n}\right) r^{n} \frac{\sin n t}{\sin t}\right]\right| \geq \frac{1-r}{4 \alpha}\left(\frac{1-r}{1+r}\right)^{\alpha}\left[1-\left(\frac{1-r}{1+r}\right)^{2 \alpha}\right] .
$$

By Theorem 1, the condition of the univalence of the new function, obtained by the variation of the $m$-th coefficient $c_{m}$ gives

$$
\left|\sum_{n=1}^{\infty}\left[\left(a_{n} z^{n}-a_{-n} \bar{z}^{n}\right) r^{n} \frac{\sin n t}{\sin t}\right]+\sigma \lambda \rho^{|m|} \frac{\sin m t}{\sin t}\right|>0, z \in \Delta \backslash 0, \quad \rho=|z|,
$$

$t \in(0, \pi / 2]$, where $|\sigma|=1$. It is fulfilled provided that

$$
\left|\lambda \frac{\sin m t}{\sin t}\right|<\frac{1-r}{4 \alpha}\left(\frac{1-r}{1+r}\right)^{\alpha}\left[1-\left(\frac{1-r}{1+r}\right)^{2 \alpha}\right]
$$

Hence it is certainly fulfilled if the inequality concerning $|\lambda|$, from the statement of Lemma, is true.

Acknowledgment. This work was supported by RFBR (project No 1401-92692 and No 14-01-00510) and by Program of Strategic Development of Petrozavodsk State University. The author would like to thank the reviewers for reading this article carefully and making valuable comments. 


\section{References}

[1] Bieberbach L. Über die Koeffizienten derjenigen Potenzreihen, welche eine schlichte Abbildung des Eincheitskreises vermitteln. S. B. Preuss. Acad. Wiss., 1916, vol. 138, pp. 940-955.

[2] de Branges L. A. Proof of the Bieberbach conjecture. Acta Math., 1985, vol. 154 , pp. $137-152$.

[3] Duren P. Harmonic mappings in the plane. Cambridge, 2004, 214 p.

[4] Clunie J., Sheil-Small T. Harmonic univalent functions. Ann. Acad. Sci. Fenn., A.1. Math, 1984, vol. 9, pp. 3-25.

[5] Ponnusamy S., Sairam Kaliraj A. On the coefficient conjecture of Clunie and Sheil-Small on univalent harmonic mappings. Proc. Indian Acad. Sci., 2014 (to appear).

[6] Bazilevich I. E. The problem of coefficients of univalent functions. Math. J. of the Aviation Institute. [Moscow], 1945, pp. 29-47. (in Russian)

[7] Bharanedhar S. V., Ponnusamy S. Coefficient conditions for harmonic univalent mappings and hypergeometric mappings. Rocky Mountain J. Math., 2014, vol. 44, no. 3, pp. 753-777.

[8] Ponnusamy S., Rasila A. Planar harmonic and quasiregular mappings. CMFT, RMS-Lecture Notes Series, 2013, no. 19, pp. 267-333.

[9] Sheil-Small T. Constants for planar harmonic mappings. J. London Math. Soc., 1990, vol. 42, pp. 237-248.

Received September 7, 2014.

In revised form, November 17, 2014.

Petrozavodsk State University

33, Lenina st., 185910 Petrozavodsk, Russia

E-mail: vstarv@list.ru 